\title{
Pediatric Burkitt's Lymphoma and Diffuse B cell Lymphoma: Are Surveillance Scans Required?
}

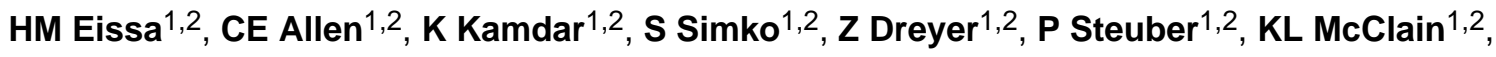 \\ RP Guillerman ${ }^{3}$, and Catherine M. Bollard ${ }^{1,2}$ \\ ${ }^{1}$ Texas Children's Cancer Center, Baylor College of Medicine, Texas Children's Hospital, \\ Houston, Texas 77030, USA \\ 2Department of Pediatrics, Baylor College of Medicine, Texas Children's Hospital, Houston, \\ Texas 77030, USA \\ ${ }^{3}$ Department of Radiology, Baylor College of Medicine, Texas Children's Hospital, Houston, \\ Texas 77030, USA
}

\begin{abstract}
Outcomes in pediatric B-Non-Hodgkin Lymphoma have improved with intensive chemotherapy protocols, with long-term survival now over $80 \%$. However, long-term adverse effects of therapy and poor outcomes for patients who relapse remain challenges. In this study, we aimed to evaluate the potential risks and benefits of routine relapse surveillance imaging after the completion of therapy. We reviewed 44 B NHL patients diagnosed and treated at Texas Children's Cancer Center in the period between 2000 to 2011. All cross-sectional diagnostic imaging examinations performed for disease assessment after completion of chemotherapy were reviewed and cumulative radiation dosage from these examinations and the frequency of relapse detection by these examinations were recorded. Only 3 patients of the 44 relapsed (6.8\%), though none of the relapses were initially diagnosed by CT or FDG-PET scans. Median effective dose of ionizing radiation per patient was $40.3 \mathrm{mSv}$ with an average of $49.1 \mathrm{mSv}$ (range 0-276 mSv). This singleinstitution study highlights the low relapse rate in pediatric B-NHL with complete response at the end of therapy, the low sensitivity of early detection of relapse with surveillance CT or FDG-PET imaging, and the costs and potential increased risk of secondary malignancies from cumulative radiation exposure from surveillance imaging. We propose that routine surveillance CT or FDGPET scans for these patients may not be necessary.
\end{abstract}

\section{INTRODUCTION}

Childhood non-Hodgkin lymphoma (NHL) has been subdivided based on treatment strategy into lymphoblastic lymphoma (LBL) of the precursor B subtype and T cell type, B-cell nonHodgkin lymphoma (B-cell NHL), and anaplastic large cell lymphoma (ALCL). B-cell nonHodgkin lymphomas in children and adolescents are comprised primarily of Burkitt lymphoma (BL), Burkitt-like lymphoma and diffuse large B-cell lymphoma (DLBCL) (1).

Corresponding author: Catherine Bollard, MD, Center for Cell and Gene Therapy, Baylor College of Medicine, 6621 Fannin Street, MC 3-3320, Houston, Tx 77030, USA, Phone: 832824 4781, Fax: 832825 4732, cmbollar@ txch.org. 
The outcome of B-cell NHL has dramatically improved over the course of the last few years with the introduction of short-term repeated intensive chemotherapy courses. The 5-year survival of pediatric B-cell NHL patients currently exceeds $80 \%$ (2).

The improved survival raises concerns about the appropriate protocol for relapse surveillance imaging in light of increasing concerns about the relative benefits, costs and long-term adverse consequences of radiation exposure (3). Cumulative radiation exposure from repetitive surveillance imaging puts long-term pediatric cancer survivors at risk for radiation-induced second malignancies $(4,5$, and 6$)$. The magnitude of the risk depends on the type and number of radiologic exams used for surveillance. Hence, the purpose of this study was to evaluate the clinical utility of surveillance imaging by estimating the cumulative radiation dose and yield of relapse detection from surveillance imaging studies in a cohort of pediatric patients with B-cell NHL.

\section{MATERIALS AND METHODS}

We identified 50 pediatric patients diagnosed with B-cell NHL (including Burkitt Lymphoma, Burkitt-like lymphoma and DLBCL) and treated at Texas Children Cancer Center during the period from 2000 through 2011. Of these, 6 patients were excluded from further analysis since they failed to achieve remission, either because of primary refractory disease or relapse before completion of chemotherapy, and therefore were never placed on standard surveillance protocols. Remission was defined as $\ 80 \%$ decrease in tumor size, defined as the product of the perpendicular diameters in the axial plane or return to normal organ or lymph node size with no extra-nodal masses, as per Children's Oncology Group $(\mathrm{COG})$ criteria. We noted the disease status and imaging studies involving ionizing radiation (radiographs, CT scans, and nuclear medicine scans including FDG-PET) of all 44 patients who were in remission after completion of their primary chemotherapy protocol until the date of their last documented visit, relapse or death, whichever occurred first. Their ages ranged from 3-18 yrs, with 7 females and 37 males, see table (1) for patient characteristics.

Radiation effective doses from CT exams were estimated from published surveys of contemporary CT clinical practice and the age-specific $50^{\text {th }}$ percentile dose level from the HMO Research Network (3). For example, the estimated per exam effective dose was $4 \mathrm{mSv}$ for chest CT and $8 \mathrm{mSv}$ for abdomen/pelvis CT for a 5-9 year-old, and $5.3 \mathrm{mSv}$ for chest $\mathrm{CT}$ and $11.1 \mathrm{mSv}$ for abdomen/pelvis CT for a 10-14 year-old. The radiation effective doses from nuclear medicine scans including FDG-PET were estimated by applying effective dose conversion factors to published pediatric radiopharmaceutical dosing guidelines from the Pediatric Nuclear Medicine Dose Reduction Workgroup (7, 8). For example, the estimated per exam effective dose for FDG-PET was $5.6 \mathrm{mSv}$ for a 5 year-old, $6.4 \mathrm{mSv}$ for a 10 yearold, and $8.6 \mathrm{mSv}$ for a 15 year-old. The estimated per exam effective doses were $25 \mathrm{mSv}$ for gallium scintigraphy and $2.8 \mathrm{mSv}$ for bone scintigraphy. The radiation doses from imaging exams with negligible radiation doses (such as chest radiographs or DXA) compared to CT or with clinical indications other than tumor relapse surveillance (such as cardiac nuclear MUGA scans) were excluded from analysis.

The study was approved by Baylor College of Medicine Institutional Review Board (IRB). 


\section{RESULTS AND DISCUSSION}

Out of the 44 patients who achieved a complete remission after receiving treatment for Bcell NHL at Texas Children's Cancer Center between the years 2000 to 2010, there were three patients who relapsed. Two patients relapsed within the first 6 months (month 2 and month 4) after completion of chemotherapy and the third patient relapsed about 4 years after completion of chemotherapy. Of the two patients who relapsed within the first 6 months, one had DLBCL and had an underlying diagnosis of ataxia-telangiectasia and the other had BL. The patient who relapsed 4 years after completion of chemotherapy had DLBCL, and in case of such late relapse it is unclear whether this represents a true relapse of the original malignancy or a second malignancy. None of the relapses were detected by surveillance CT, FDG-PET or gallium scintigraphy prior to clinical suspicion of relapse.. Suspicion of relapse was prompted by progressive lymph node enlargement noted on physical exam in one case, by worsening cough in a second case, and by ascites noted on abdominal ultrasonography done as surveillance in a third case who was otherwise asymptomatic. One patient who did not relapse underwent 3 FDG-PET scans showing increased FDG uptake suspicious for relapse. However, relapse was not confirmed on follow-up CT scans. This underscores that one of the detriments of routine surveillance imaging in the absence of clinical suspicion of relapse is false positive examinations leading to further unnecessary examinations. In addition, the adverse psychological impact on the patient and patient's family should not be disregarded when such uncertainty regarding their disease status is created.

During the surveillance period, the patients received a total of 480 imaging examinations (mean 10.9 scan per patient, range 1-71), consisting of 185 radiographs, $87 \mathrm{CT}$ scans of the chest, $110 \mathrm{CT}$ scans of the abdomen/pelvis, $37 \mathrm{CT}$ scans of the neck, $27 \mathrm{CT}$ scans of other body regions, 24 Gallium scans and 8 FDG-PET/CT scans. Radiographs were excluded from the calculations as they represent negligible radiation exposure compared to $\mathrm{CT}$, gallium and FDG-PET scans. Imaging modalities such as DXA scans, nuclear medicine glomerular filtration rate (GFR) scans, nuclear medicine gastric emptying studies, nuclear medicine cardiac MUGA (multigated acquisition) scans, and nuclear medicine bone scans not obtained for relapse surveillance were excluded. Excluding the latter tests, patients in this study incurred a mean radiation effective dose of $49.11 \mathrm{mSv}$ and median radiation effective dose of $40.3 \mathrm{mSv}$ per patient (range 0-276). These are in the range of radiation doses from CT scans in childhood that have been associated with an increased risk of subsequent malignancy. For example, in a recent retrospective cohort study, children who were exposed to a cumulative dose of at least $30 \mathrm{mGy}$ of radiation from CT were approximately three times more likely to develop leukemia (9).

Our study is the first that has specifically evaluated the utility of surveillance imaging for clinically occult relapse in pediatric patients with B-cell NHL. Study limitations include its retrospective nature and the small number of relapses that limits the statistical power and precision for determining the efficacy of surveillance imaging for relapse detection. In addition, we could not review imaging examinations that were performed during the surveillance period outside of our institution. 
Potential carcinogenic effect of radiation from diagnostic imaging has been a topic of rising interest, especially in children who are believed to be more at risk compared to adults. With improved survival rates, many pediatric cancers patients are anticipated to have a longer life expectancy allowing more time for secondary malignancies to develop. Although the increase in second malignancy risk from radiation exposure from surveillance imaging scans for individual patients may be slight in comparison to the risk from other sources such as radiotherapy, even this slight risk should not be overlooked in consideration of the questionable clinical benefit of these surveillance scans. Revision of current pediatric cancer protocols to incorporate more judicious use of imaging surveillance is needed (5). As an alternative to current surveillance protocols stipulating numerous CT scans over a several year period, we believe that abdominal ultrasonography and chest radiographs are generally sufficient to follow sites of abdominal or mediastinal tumor involvement. Routine CT or FDG-PET/CT surveillance imaging of these children in the absence of symptoms or signs of relapse would expose them to radiation without clear benefit and therefore we recommend that surveillance imaging is not warranted in these patient. It will however be very important to conduct multi-institutional studies involving larger patient cohorts to optimize the longterm follow-up strategies for these patients.

\section{References}

1. Reiter A. Diagnosis and treatment of childhood non-Hodgkin lymphoma. Hematology Am Soc Hematol Educ Program 2007. 2007:285-296.10.1182/asheducation-2007.1.285

2. Bollard CM, Lim MS, Gross TG. COG Non-Hodgkin Lymphoma Committee. Children's Oncology Group's 2013 blueprint for research: Non-Hodgkin lymphoma. Pediatr Blood Cancer. 2013 Jun; 60(6):979-84. Epub 2012 Dec 19. 10.1002/pbc.24416 [PubMed: 23255391]

3. Miglioretti DL, Johnson E, Williams A, Greenlee RT, Weinmann S, Solberg LI, Feigelson HS, Roblin D, Flynn MJ, Vanneman N, Smith-Bindman R. The use of computed tomography in pediatrics and the associated radiation exposure and estimated cancer risk. JAMA Pediatr. 2013 Jun. 10:1-8. Epub ahead of print. 10.1001/jamapediatrics.2013.311

4. Baysson H, Etard C, Brisse HJ, et al. Diagnostic radiation exposure in children and cancer risk: current knowledge and perspectives. Arch Pediatr. 2012; 19:64-73. [PubMed: 22130615]

5. Rathore N, Eissa HM, Margolin JF, Liu H, Wu MF, Horton T, Kamdar K, Dreyer Z, Steuber P, Rabin KR, Redell M, Allen CE, McClain KL, Guillerman RP, Bollard CM. Pediatric Hodgkin Lymphoma: Are We Over-Scanning Our Patients? Pediatr Hematol Oncol. 2012 Aug; 29(5):41523. Epub 2012 May 25. 10.3109/08880018.2012.684198 [PubMed: 22632168]

6. Voss SD, Chen L, Constine LS, et al. Surveillance Computed Tomography Imaging and Detection of Relapse in Intermediate- and Advanced-Stage Pediatric Hodgkin's Lymphoma: A Report From the Children's Oncology Group. J Clin Oncol. 2012; 30(21):2635-2640. [PubMed: 22689804]

7. Gelfand MJ. Dosimetry of FDG PET/CT and other molecular imaging applications in pediatric patients. Pediatr Radiol. 2009; 39 (Suppl 1):S46-S56. [PubMed: 19083225]

8. Treves ST, Parisi MT, Gelfand MJ. Pediatric radiopharmaceutical doses: new guidelines. Radiology. 2011; 261:347-349. [PubMed: 22012901]

9. Pearce MS, Salotti JA, Little MP, McHugh K, Lee C, Kim KP, Howe NL, Ronckers CM, Rajaraman P, Sir Craft AW, Parker L, Berrington de González A. Radiation exposure from CT scans in childhood and subsequent risk of leukaemia and brain tumors: a retrospective cohort study. Lancet. 2012; 380(9840):499-505. Epub 2012 Jun 7. 10.1016/S0140-6736(12)60815-0 [PubMed: 22681860] 
TABLE 1

Patients Characteristics

\begin{tabular}{|c|c|c|c|c|}
\hline Diagnosis & Age in yrs & Sex & Time to relapse in months & Total radiation $(\mathrm{mSV})$ \\
\hline Burkitt & 6.00 & M & 0 & 99 \\
\hline Burkitt & 9.00 & M & 0 & 12.8 \\
\hline Burkitt & 9.00 & M & 0 & 49 \\
\hline Burkitt & 10.50 & $\bar{M}$ & 2 & 75.7 \\
\hline Burkitt & 12.00 & M & 0 & 51.2 \\
\hline Burkitt & 3.00 & M & 0 & 20 \\
\hline Burkitt & 10.00 & $\mathrm{M}$ & 0 & 24.8 \\
\hline Burkitt-like & 4.50 & M & 0 & 132 \\
\hline Burkitt & 12.50 & M & 0 & 90.6 \\
\hline Burkitt & 9.50 & $M$ & 0 & 25 \\
\hline Burkitt & 3.50 & $\mathrm{M}$ & 0 & 14 \\
\hline Burkitt & 6.00 & M & 0 & 40 \\
\hline Burkitt & 6.00 & M & 0 & 20 \\
\hline Burkitt & 7.00 & $\bar{F}$ & 0 & 81 \\
\hline Burkitt & 7.00 & $\mathrm{M}$ & 0 & 54 \\
\hline Burkitt & 8.50 & M & 0 & 0 \\
\hline Burkitt & 14.00 & $M$ & 0 & 123.4 \\
\hline Burkitt & 3.50 & $\mathrm{M}$ & 0 & 40 \\
\hline Burkitt & 5.50 & $M$ & 0 & 48 \\
\hline Burkitt & 5.00 & $M$ & 0 & 48 \\
\hline Burkitt & 11.50 & $\mathrm{~F}$ & 0 & 49.7 \\
\hline Burkitt & 8.50 & M & 0 & 16 \\
\hline Burkitt & 6.00 & $\mathrm{M}$ & 0 & 64 \\
\hline Burkitt & 13.50 & $M$ & 0 & 32.8 \\
\hline Burkitt & 5.00 & M & 0 & 24 \\
\hline Burkitt & 5.00 & $M$ & $\overline{0}$ & 13.6 \\
\hline Burkitt & 8.00 & $\mathrm{M}$ & 0 & 12 \\
\hline Burkitt & 16.00 & M & 0 & 11.1 \\
\hline Burkitt Leukemia & 15.50 & $M$ & 0 & 92 \\
\hline Burkitt Leukemia & 17.00 & $M$ & 0 & 18.4 \\
\hline DLBCL & 18.00 & M & 4 & 40.6 \\
\hline DLBCL & 11.50 & $\mathrm{M}$ & 0 & 33.3 \\
\hline DLBCL & 5.00 & M & 0 & 24 \\
\hline DLBCL & 4.00 & $\mathrm{~F}$ & 0 & 0 \\
\hline DLBCL & 15.00 & $\mathrm{~F}$ & 0 & 276 \\
\hline DLBCL & 14.00 & $\mathrm{M}$ & 47 & 104.5 \\
\hline DLBCL & 7.50 & $\mathrm{~F}$ & 0 & 44 \\
\hline
\end{tabular}




\begin{tabular}{|l|l|l|l|r|}
\hline Diagnosis & Age in yrs & Sex & Time to relapse in months & Total radiation (mSV) \\
\hline DLBCL & 16.00 & $\mathrm{M}$ & 0 & 45.9 \\
\hline DLBCL & 11.00 & $\mathrm{M}$ & 0 & 16.4 \\
\hline DLBCL & 11.00 & $\mathrm{~F}$ & 0 & 53.5 \\
\hline DLBCL & 11.00 & $\mathrm{~F}$ & 0 & 59.8 \\
\hline DLBCL & 3.50 & $\mathrm{M}$ & 0 & 25 \\
\hline DLBCL & 13.50 & $\mathrm{M}$ & 0 & 10.6 \\
\hline DLBCL & 9.00 & $\mathrm{M}$ & 0 & 45.2 \\
\hline
\end{tabular}

